\title{
Comparison of Chebyshev and Legendre Polynomial Expansion of Phase Function of Cloud and Aerosol Particles
}

\author{
Feng Zhang, ${ }^{1,2}$ Kun Liu, ${ }^{3}$ Quan Yang, ${ }^{1}$ Kun Wu, ${ }^{1}$ and Jian-Qi Zhao ${ }^{4}$ \\ ${ }^{1}$ Key Laboratory of Meteorological Disaster, Ministry of Education (KLME)/Joint International Research Laboratory of Climate and \\ Environment Change (ILCEC)/Collaborative Innovation Center on Forecast and Evaluation of Meteorological Disaster (CICFEMD), \\ Nanjing University of Information Science and Technology, Nanjing 210044, China \\ ${ }^{2}$ State Key Laboratory of Severe Weather, Chinese Academy of Meteorological Sciences, Beijing 100081, China \\ ${ }^{3}$ Shenzhen National Climate Observatory, Shenzhen 518040, China \\ ${ }^{4}$ State Key Laboratory of Numerical Modeling for Atmospheric Sciences and Geophysical Fluid Dynamics, \\ Institute of Atmospheric Physics, Chinese Academy of Sciences, Beijing 100029, China
}

Correspondence should be addressed to Jian-Qi Zhao; zhaojianqi@mail.iap.ac.cn

Received 26 May 2017; Accepted 30 July 2017; Published 18 September 2017

Academic Editor: Jia Yue

Copyright (C) 2017 Feng Zhang et al. This is an open access article distributed under the Creative Commons Attribution License, which permits unrestricted use, distribution, and reproduction in any medium, provided the original work is properly cited.

\begin{abstract}
Chebyshev and Legendre polynomial expansion is used to reconstruct the Henyey-Greenstein phase function and the phase functions of spherical and nonspherical particles. The result of Legendre polynomial expansion is better than that of Chebyshev polynomial for around 0-degree forward angle, while Chebyshev polynomial expansion produces more accurate results in most regions of the phase function. For large particles like ice crystals, the relative errors of Chebyshev polynomial can be two orders of magnitude less than those of Legendre polynomial.
\end{abstract}

\section{Introduction}

The dynamics and transmission of the atmosphere rely on the distribution and magnitude of the net radiative heating of the atmosphere system. In the stratosphere, the net radiative heating depends solely on the imbalance between infrared radiative loss and local absorption of solar UV radiation [1]. The distribution of the radiative sources and sinks exerts a zero order control on the large-scale seasonally varying zonal wind fields and mean temperature in the stratosphere [2]. It is known that the large-scale circulation in the stratosphere is different from that in the troposphere, and eddies are as elementary to the circulation as the differential solar radiative heating. Radiative processes play a key role in driving global climate change and establishing temperature structure of the atmosphere [3]. Phase function always displays very intricate structures, as the peak value of the forward scattering could be several orders of magnitude larger than that of the back scattering. Currently, Legendre polynomial expansion is widely used in representing the scattering phase function and it is sensitive to the forward scattering peak of phase function. Thus, the Legendre polynomial series converge very slowly; it could take literally thousands of Legendre polynomial terms to reconstruct the original phase function.

In order to improve the parameterization of phase function, several techniques have been developed such as the $\delta$ - $M$ method [4], the $\delta$-fit method [5], GT approximation in geometrical truncation [6], MRTD (multiresolution time domain) scattering model [7, 8], Q-space analysis [9], and invariant imbedding T-matrix method [10]. These techniques tend to remove the strong forward scattering peak instead of seeking a fast convergence expression of phase function. Even if the strong forward scattering peak has been removed by the above techniques, the phase function itself still needs to be parameterized with limited terms of Legendre polynomial expansion. How to represent the scattering phase function accurately and efficiently is the goal of this study. In the following, various phase functions are expanded by Legendre polynomial and the second kind of Chebyshev polynomial. In Section 3, the accuracies of the scattering 
phase functions reconstructed by Chebyshev and Legendre polynomial expansions are discussed. A short summary is given in Section 4.

\section{Theoretical Background}

The scattering phase function using Legendre polynomial expansion can be written as

$$
P(\cos \Theta)=\sum_{n=0}^{N} \omega_{n} P_{n}(\cos \Theta),
$$

where $P_{n}$ and $\Theta$ are the Legendre function and the scattering angle, respectively. $N$ is the number of expansion terms. $\omega_{n}$, determined from the orthogonal property of Legendre polynomial, can be written as

$$
\omega_{n}=\frac{2 n+1}{2} \int_{-1}^{1} P(\cos \Theta) P_{n}(\cos \Theta) d \cos \Theta,
$$

where $\omega_{0}=1, \omega_{1}=3 g$, and $g$ is the asymmetry factor.

Using the second kind of Chebyshev function, the phase function can be expanded as

$$
P(\cos \Theta)=\sum_{n=0}^{N} \chi_{n} U_{n}(\cos \Theta),
$$

where $\Theta$ is the scattering angle and $U_{n}$ is the second kind of Chebyshev function. $U_{0}=1, U_{1}=2 x, U_{2}=4 x^{2}-1$. The second kind of Chebyshev polynomial $U_{n}$ of degree $n$ is

$$
U_{n}(x)=\sum_{k=0}^{n / 2}(-1)^{k} \frac{(n-k) !}{k !(n-2 k) !}(2 x)^{n-2 k} .
$$

There is a recurrence formula for $U_{n}$

$$
U_{n+1}(x)+U_{n-1}(x)=2 x U_{n}(x) .
$$

The second kind of Chebyshev series is orthogonal polynomial with respect to the weighting function $\sqrt{1-x^{2}}$

$$
\int_{-1}^{1} \sqrt{1-x^{2}} U_{m}(x) U_{n}(x) d x=\frac{1}{2} \pi \delta_{n m}
$$

where

$$
\delta_{n, m}= \begin{cases}1 & (n=m) \\ 0 & (n \neq m),\end{cases}
$$

is the Kronecker delta.

Similarly, $\chi_{n}$ can be written from the orthogonal property of the second kind of Chebyshev polynomial in the form

$$
\chi_{n}=\frac{2}{\pi} \int_{-1}^{1} \sqrt{1-\cos ^{2} \Theta} P(\cos \Theta) U_{n}(\cos \Theta) d \cos \Theta .
$$

\section{Comparison of Phase Function}

In this section, the phase function expansions by the Legendre and the second kind of Chebyshev polynomials are compared for accuracy and efficiency. The samples will be taken from Henyey-Greenstein (HG) phase function and the phase functions of spherical and nonspherical particles.

3.1. Henyey-Greenstein Phase Function. The HG function [11] has a remarkable analytical property as

$$
P_{\mathrm{HG}}(\cos \Theta)=\frac{1-g^{2}}{\left(1-2 g \cos \Theta+g^{2}\right)^{3 / 2}},
$$

where $g$ is the asymmetry factor. The phase functions reconstructed in various terms of Chebyshev and Legendre polynomials against the benchmark results of HG phase functions are shown in Figure 1. In Figure 1(a), the phase functions reconstructed by Chebyshev and Legendre polynomials are shown for 8 terms (top panel), 16 terms (middle panel), and 24 terms (bottom panel); in Figure 1(b), the relative errors of phase function are shown.

It can be seen in Figure 1 that the reconstructed phase function becomes more accurate with the increasing of terms. On the whole, the results of Chebyshev polynomial expansion are more accurate compared to the Legendre polynomial expansion between 12 degrees and 168 degrees. In addition, Chebyshev polynomial provides better results at large scattering angles. On the contrary, Legendre polynomial has shown good results at forward (0-degree) and back (180-degree) angles.

3.2. Phase Function of Spherical Particles. For particles of equivalent spheres, Mie theory is primarily used to calculate the single-scattering properties. In the following, the phase functions of soot and water cloud at a wavelength of $\lambda=$ $0.55 \mu \mathrm{m}$ are shown.

3.2.1. Soot. The radiative impact of soot is size distribution dependent. The soot size distribution is used to be described by the log-normal distribution

$$
n(r)=\frac{1}{r \sigma_{g} \sqrt{2 \pi}} \exp \left[-\frac{\ln ^{2}\left(r / r_{g}\right)}{2 \sigma_{g}^{2}}\right],
$$

where $r_{g}=52 \mathrm{~nm}$ and $\sigma_{g}=0.42$, which are obtained by fitting measurements of diesel soot emissions [12]. The phase function for the ensemble system is given by

$$
P(\cos \Theta)=\frac{\left(\lambda^{2} / 2 \pi^{2}\right) \int\left(i_{1}(\Theta, r)+i_{2}(\Theta, r)\right) n(r) d r}{\int Q_{\text {sca }}(r) r^{2} n(r) d r},
$$

where $Q_{\text {sca }}$ is the scattering efficiency, $i_{1}$ is the square of vertical scattering amplitudes, and $i_{2}$ is the square of horizontal scattering amplitudes. The asymmetry factor of soot $g$ is 0.53 at wavelength $\lambda=0.55 \mu \mathrm{m}$.

Figure 2 shows the results of soot. The accuracy of the Chebyshev polynomial expansion is slightly better than that of the Legendre polynomial expansion in a 60-120-degree scattering angle. Because of the small size of soot particles, the phase functions reconstructed by 8 terms of Chebyshev and Legendre polynomials are both very close to the benchmark value of phase function. 

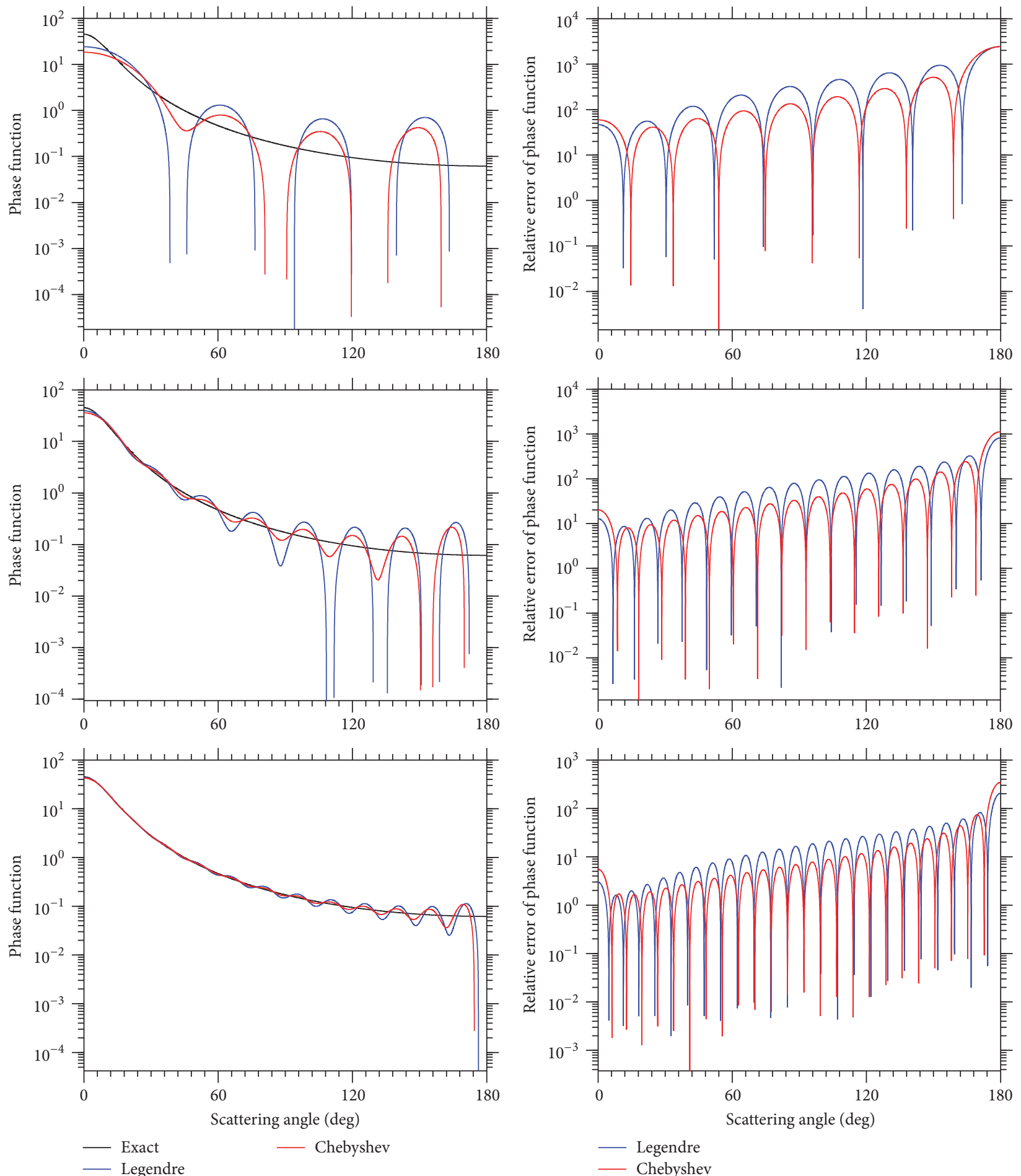

(a)

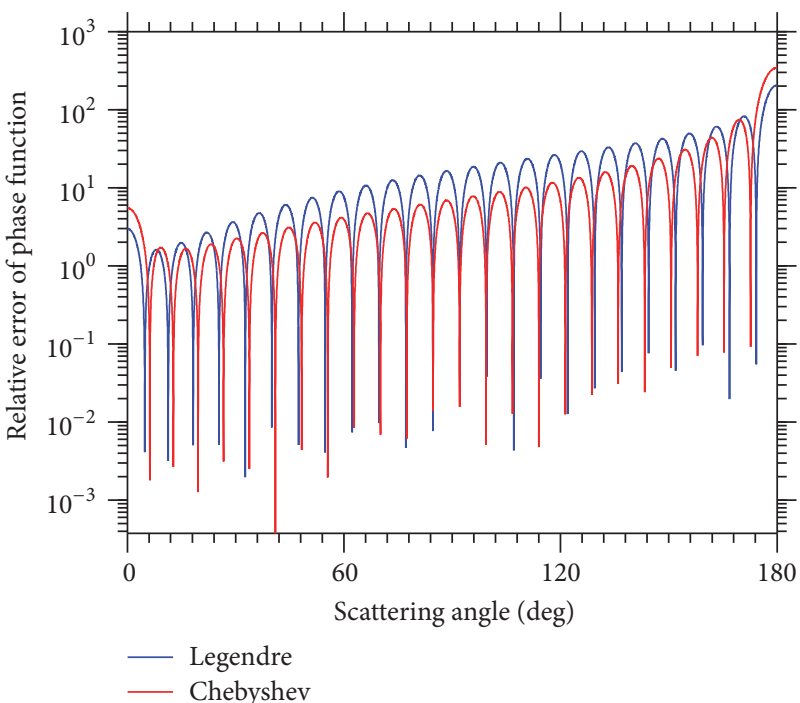

(b)

FIGURE 1: HG phase function (a) with $g=0.8$ and relative error of phase function (b) in 8,16 , and 24 terms. 


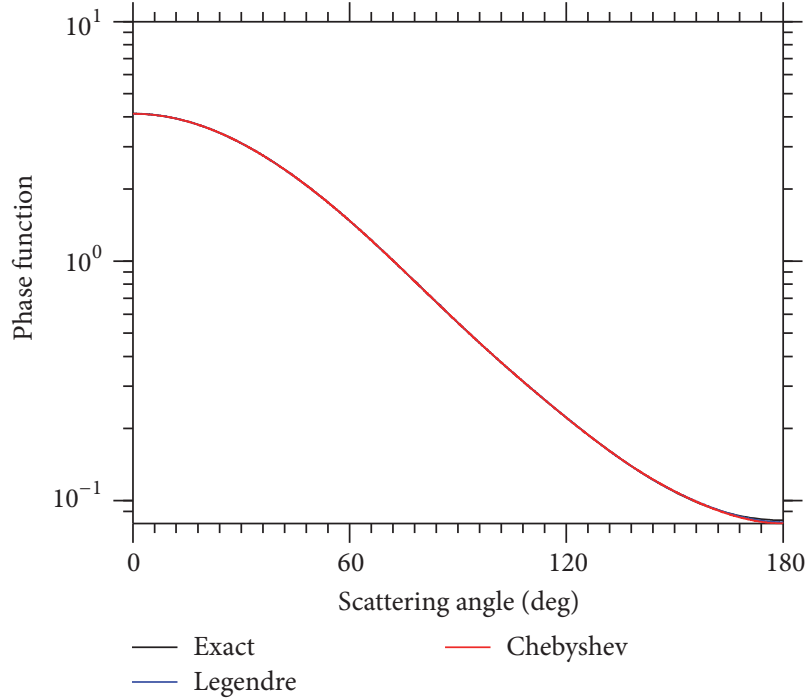

(a)

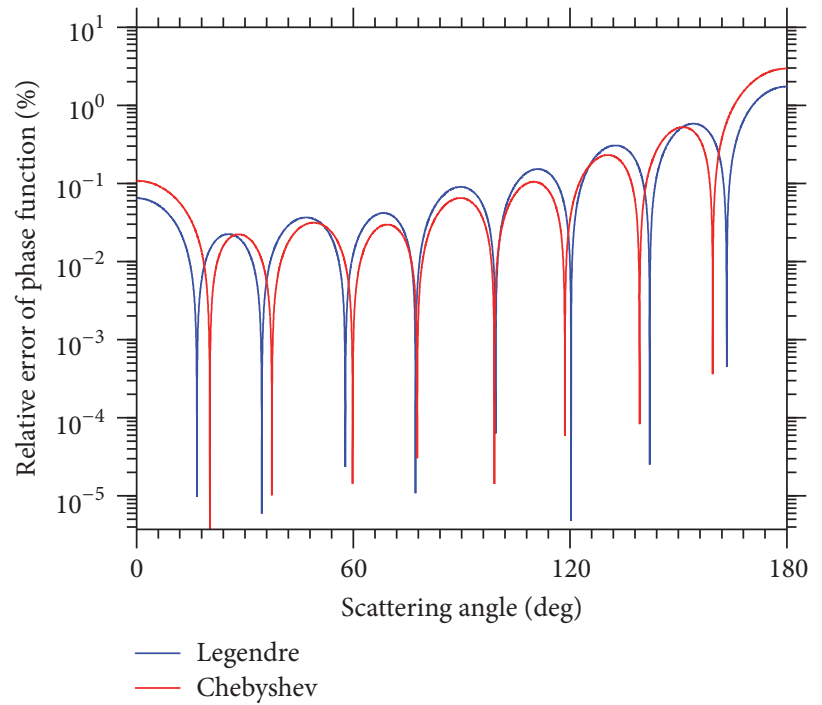

(b)

FIgURE 2: Phase function of soot (a) and relative error of phase function (b) in 8 terms.

3.2.2. Water Cloud. The size distribution of water cloud droplets tends to closely resemble gamma distributions [13] as

$$
n(r)=A r^{\alpha} e^{-\beta r}
$$

where $r$ is the radius of the water cloud and $A, \alpha$, and $\beta$ are constants. The effective radius and effective variance are $r_{e}=(\alpha+3) / \beta$ and $v_{e}=1 /(\alpha+3)$, respectively. We adopted $v_{e}=0.172$ as a constant value in cloud optical property parameterizations [14]. The effective radius is set as $5.89 \mu \mathrm{m}$, with asymmetry factor $g$ being 0.85 .

Figure 3 shows the phase function for water cloud. The relative errors of Legendre polynomial can be up to $10,000 \%$ between the scattering angles of 60 degrees and 180 degrees, and while the relative errors of Chebyshev polynomial are about one order of magnitude less than those of Legendre polynomial in 24 terms, however, the Legendre polynomial gives better results at small scattering angle.

Figure 4 shows the results of aerosol internally mixed with water cloud. In this case, the water cloud particle is the same as that in Figure 3 and the concentration of black carbon aerosol has been considered as 5000 ppb (parts per billion). It can be seen that the patterns of phase function and relative errors are similar to the results of pure water cloud.

3.3. Phase Function of Nonspherical Particle. The shapes of dust and ice cloud particles are exclusively nonspherical. It is well known that the single-scattering properties of these particles are vastly different from those spherical particles. The scattering for nonspherical particles can be calculated by finite-difference time domain method [15], bridging technique [16], T-matrix method [17], and the improved geometric optics method [18].
3.3.1. Dust Aerosol Phase Function. For dust aerosols, the radiative forcing depends strongly on their size distribution and the log-normal distribution is usually used

$$
n(r)=\frac{1}{r} \frac{N}{\sqrt{2 \pi} \ln \sigma_{g}} \exp \left[-\frac{1}{2}\left(\frac{\ln r-\ln r_{g}}{\ln r_{g}}\right)^{2}\right]
$$

where $r$ and $N$ are the radius of the equivalent sphere, with the same volume as a spheroid, and the number density of dust aerosols, respectively. $\sigma_{g}$ and $r_{g}$ are the standard deviation and mode radius, respectively. The effective radius and the effective variance are $r_{e}=r_{g} \exp \left(5 \ln ^{2} \sigma_{g} / 2\right)$ and $v_{e}=$ $\exp \left(\ln ^{2} \sigma_{g}\right)-1$, respectively. Dust particles are assumed to be spheroids $[19,20]$ with an aspect ratio of $1.7[21,22] . \sigma_{g}=2.15$ and $r_{g}=1.90 \mu \mathrm{m}$ are set for dust coarse mode. The singlescattering properties of dust aerosols are from a dust database [23], which is calculated by the combination of the T-matrix and improved geometric optics method (IGOM) [24, 25]. The asymmetry factor $g$ is 0.81 for coarse mode of dust at the wavelength of $\lambda=0.55 \mu \mathrm{m}$.

Figure 5 shows the results of phase function for coarse mode of dust. In the cases of 16- and 24-term reconstruction, both Chebyshev and Legendre polynomials can hardly fit the forward scattering peak well. In comparison with Legendre polynomial expansion, the results of Chebyshev polynomial expansion are much more accurate except for the forward scattering phase function, as the relative errors are reduced by one order of magnitude.

3.3.2. Ice Cloud Phase Function. Ice crystals with a hexagonal prism are selected for our study. In order to characterize the size distribution of ice cloud, a mean effective size as the ratio 

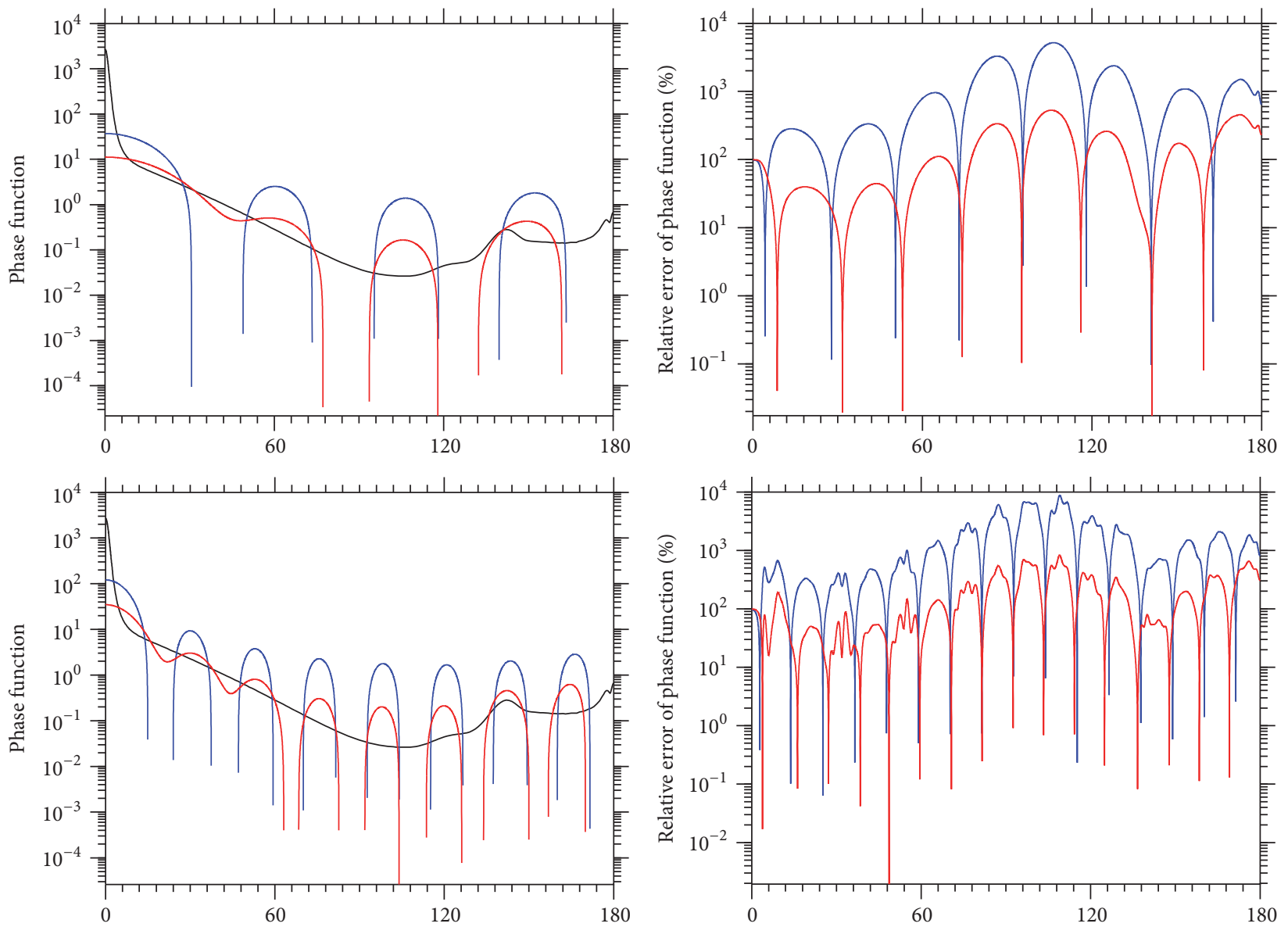

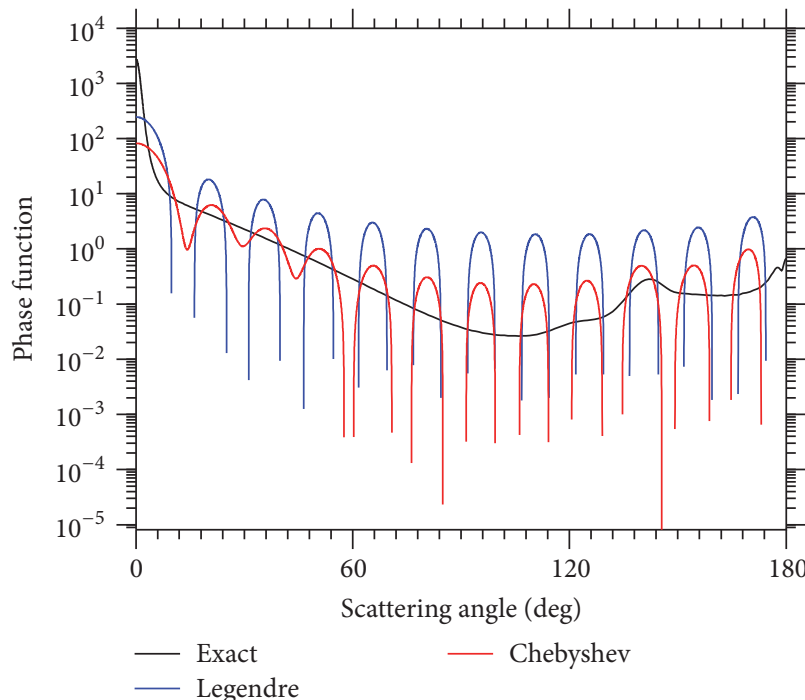

(a)

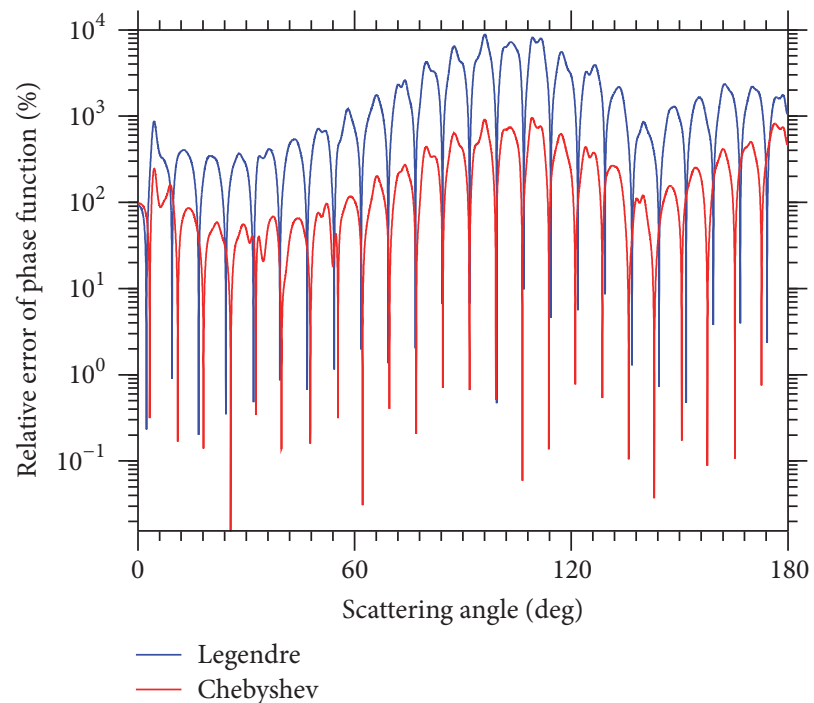

(b)

FIGURE 3: Phase function of water cloud (a) and relative error of phase function (b) in 8, 16, and 24 terms.

of ensemble particle volume and the particle projected area can be written as $[26,27]$

$$
D_{e}=\frac{3}{2} \frac{\int V(L) n(L) d L}{\int A(L) n(L) d L},
$$

where $V(L)$ and $A(L)$ are the volume and projected area, respectively, $L$ is a maximum dimension of an ice crystal, and $n(L) d L$ denotes the number density of ice crystals in the size interval $(L, L+d L)$. The single-scattering properties are from a database of ice crystals [28], which is calculated based on 

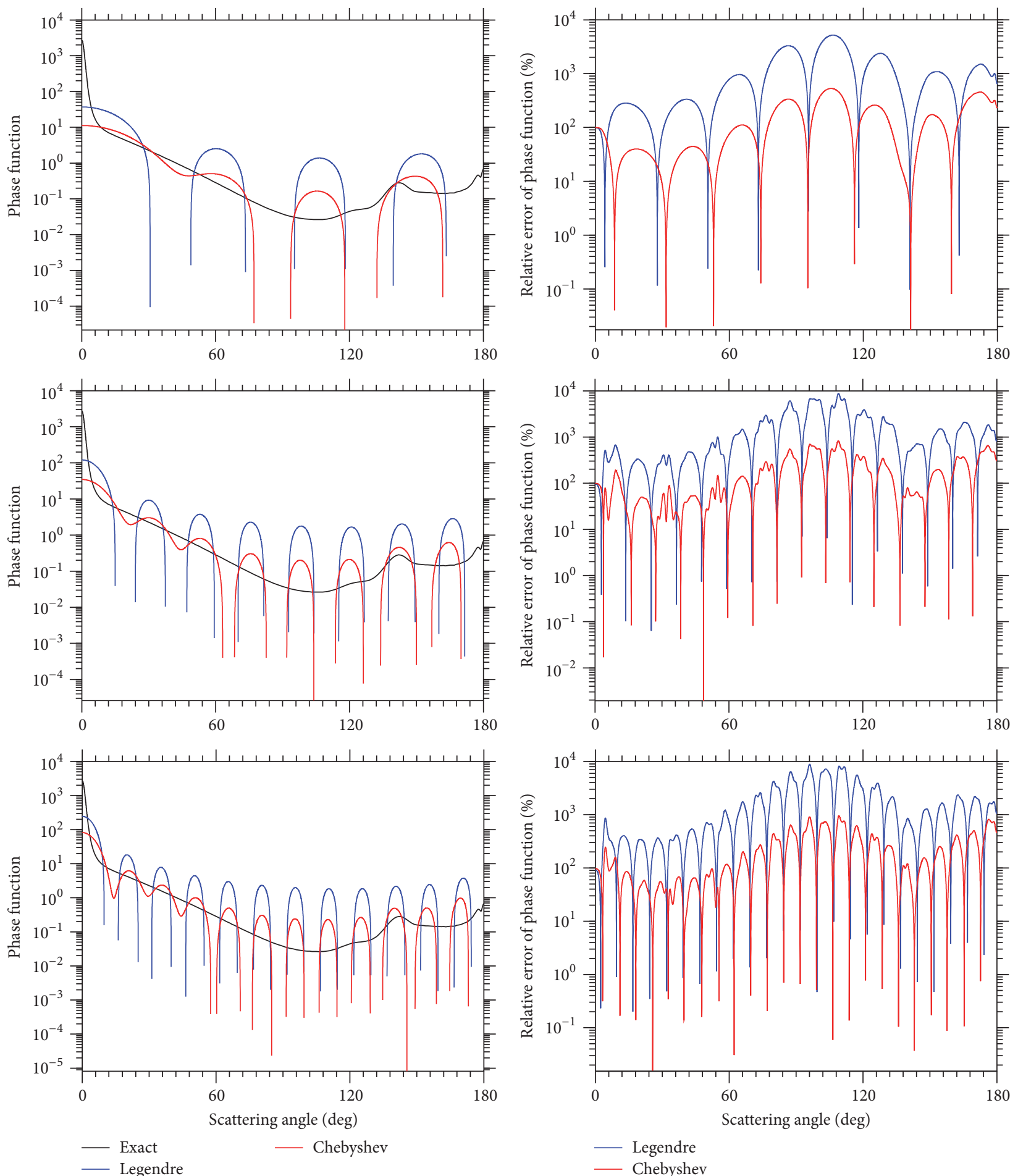

(a)

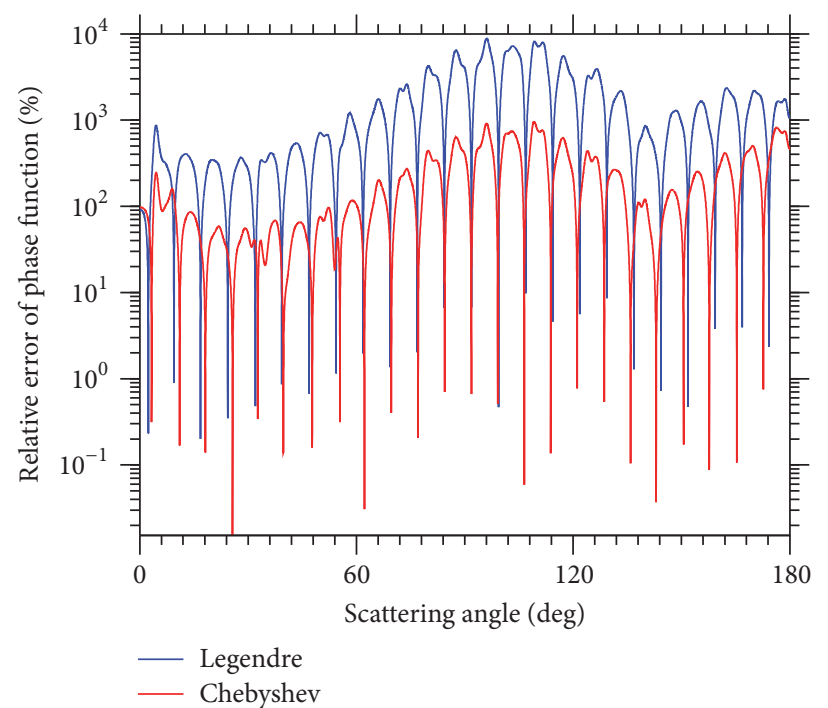

(b)

FIGURE 4: Phase function of black carbon internally mixed with water cloud (a) and relative error of phase function (b) in 8,16 , and 24 terms.

a combination of the T-matrix method, the improved geometric optics method (IGOM), and the Amsterdam discrete dipole approximation (ADDA). The asymmetry factor of ice cloud is $g=0.786$ at the wavelength of $\lambda=0.55 \mu \mathrm{m}$.
Figure 6 shows the phase function of ice cloud with $D e=$ $50 \mu \mathrm{m}$. Because of the strong forward scattering peak, the relative errors by Legendre polynomial expansion are up to $10,000 \%$, while the accuracy of phase function is dramatically 

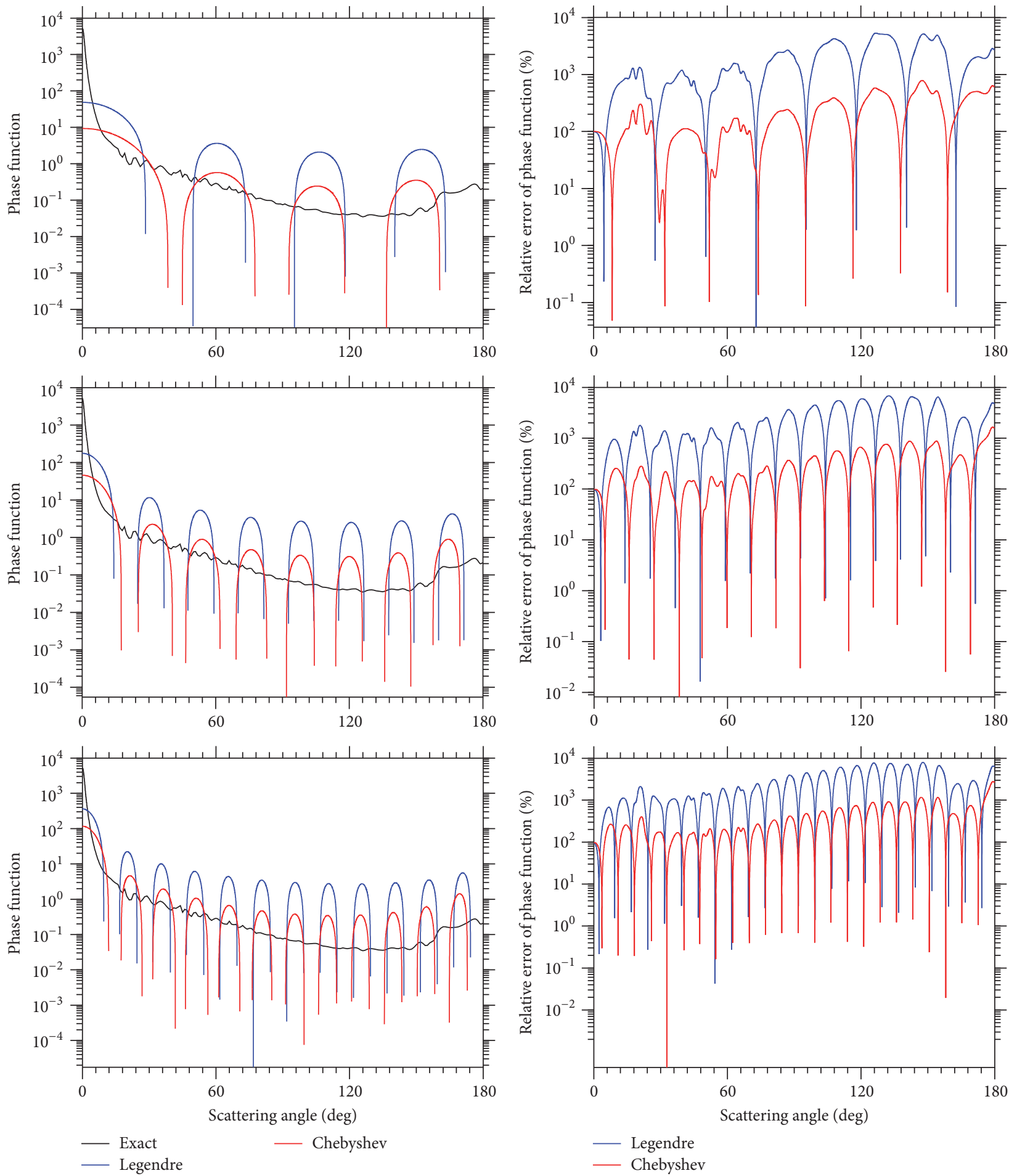

(a)

(b)

Figure 5: Phase function of coarse mode of dust (a) and relative error of phase function (b) in 8, 16, and 24 terms. 

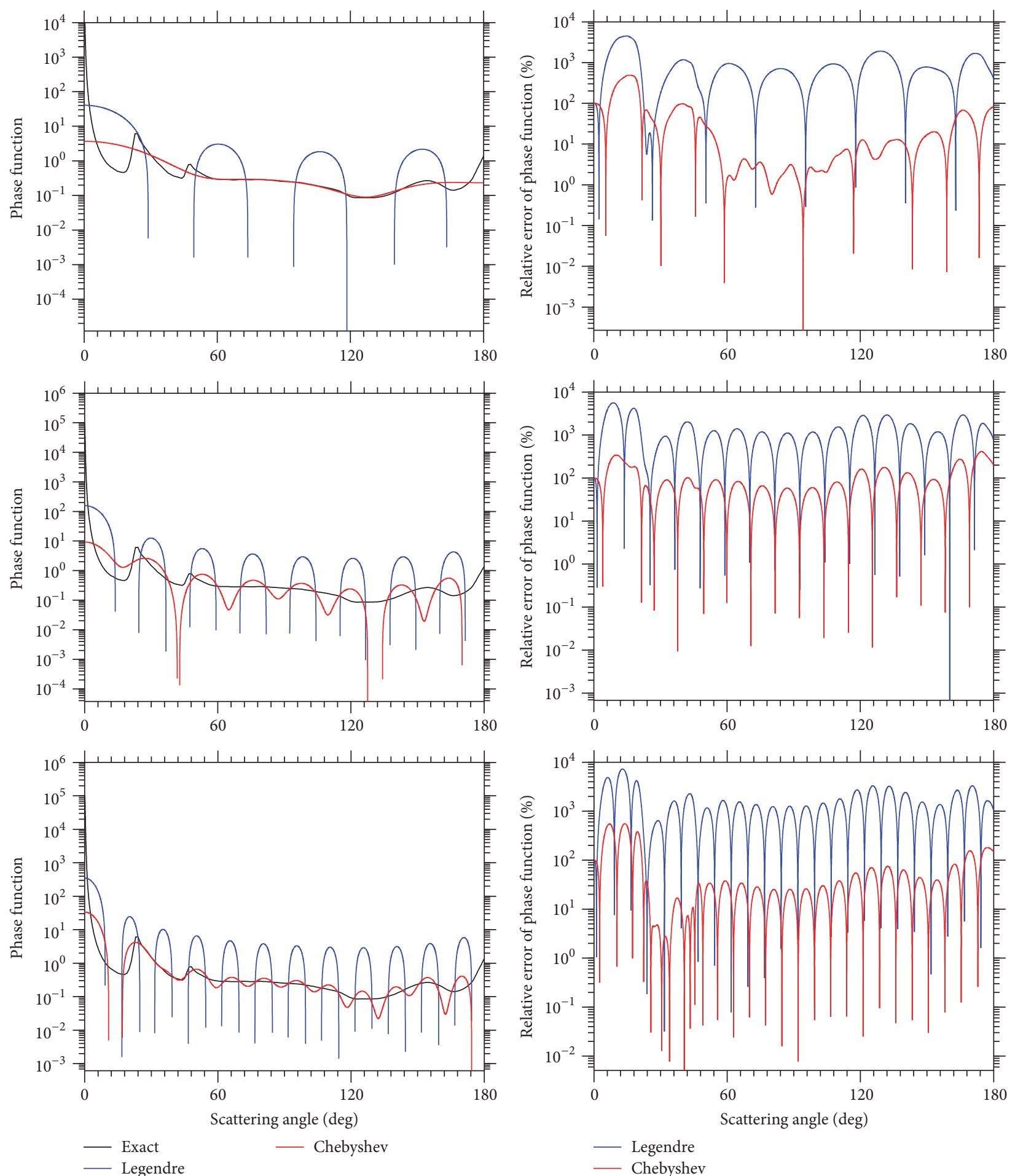

(a)

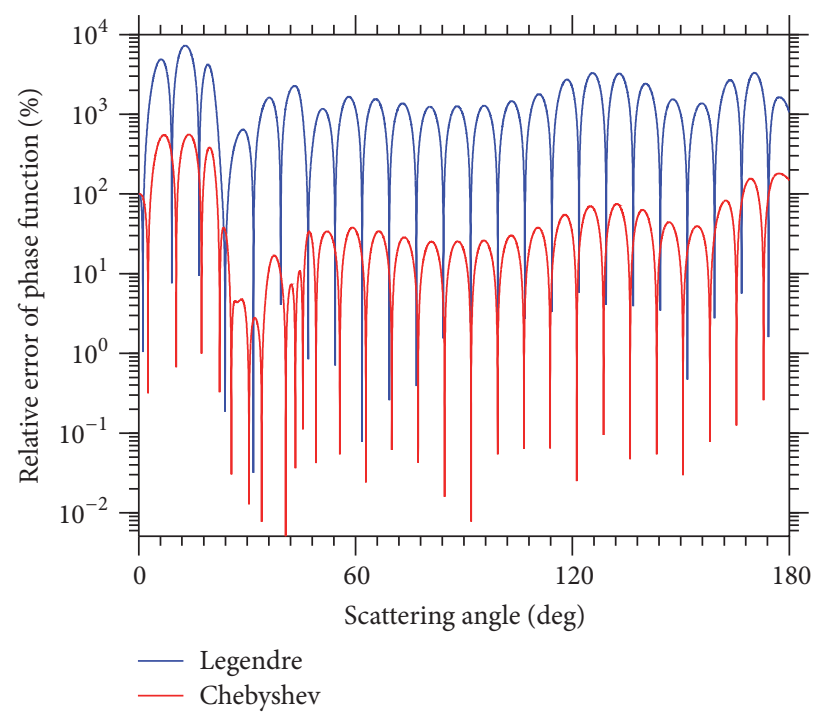

(b)

FIGURE 6: Phase function of ice cloud (a) and relative error of phase function (b) in 8, 16, and 24 terms. 
improved by using Chebyshev polynomial expansion which has been two orders of magnitude improvement in relative errors except for the small scattering angle.

\section{Summary}

In scattering and radiative transfer calculations, the complicated phase functions have to be represented by certain polynomial expansions. The second kind of Chebyshev polynomial is proposed to approximate the scattering phase functions. Compared with Legendre polynomial expansion in the scattering cases of HG, spherical and nonspherical particles, Chebyshev polynomial expansion shows more accurate results at a wide range of scattering angles, especially for large particles like ice crystals, where the relative errors are reduced by two orders of magnitude, while in the forward peak of the phase function, the Legendre polynomial produces better results than Chebyshev polynomial. It is concluded in this short note that Chebyshev polynomial is a better choice at a wide range of scattering angles. Besides, the Legendre polynomial always has better results in the forward scattering angles. In further research, $\delta$-fit or $\delta$ - $M[4,5]$ should be used in the calculation to produce more accurate results.

\section{Conflicts of Interest}

The authors declare that they have no conflicts of interest.

\section{Acknowledgments}

This work is funded by the National Key R\&D Program of China (Grant no. 2017YFA0603503), the National Natural Science Foundation of China (Grants nos. 41675003 and 41590875), the Open Project of Severe Weather (Grant no. 2016LASW-B07), and the Priority Academic Program Development (PAPD) of Jiangsu Higher Education Institutions. The ESMC contribution number is ESMC 186.

\section{References}

[1] K. Mohanakumar, Stratosphere Troposphere Interactions, Springer, Amsterdam, Netherlands, 2008.

[2] D. G. Andrews, J. R. Holton, and C. B. Leovy, Middle Atmosphere Dy-namics, Academic Press, 1987.

[3] V. Eyring, T. G. Shepherd, and D. W. Waugh, "Sparc ccmval report on the evaluation of chemistry-climate models," SPARC Report No 5, WCRP-X, WMO/TD-No X, 2010.

[4] W. Wiscombe, "The delta-m method: Rapid yet accurate radiative ux calculations," Journal of the Atmospheric Sciences, vol. 34, pp. 1408-1422, 1977.

[5] Y.-X. Hu, B. Wielicki, B. Lin et al., " $\delta$-Fit: A fast and accurate treatment of particle scattering phase functions with weighted singular-value decomposition least-squares fitting," Journal of Quantitative Spectroscopy and Radiative Transfer, vol. 65, no. 4, pp. 681-690, 2000.

[6] H. Iwabuchi and T. Suzuki, "Fast and accurate radiance calculations using truncation approximation for anisotropic scattering phase functions," Journal of Quantitative Spectroscopy and Radiative Transfer, vol. 110, no. 17, pp. 1926-1939, 2009.
[7] S. Hu, T. Gao, H. Li, M. Chen, F. Zhang, and B. Yang, "Simultaneously simulating the scattering properties of nonspherical aerosol particles with different sizes by the MRTD scattering model," Optics Express, vol. 25, no. 15, pp. 17872-17891, 2017.

[8] S. Hu, T. Gao, H. Li et al., "Application of Convolution Perfectly Matched Layer in MRTD scattering model for non-spherical aerosol particles and its performance analysis," Journal of Quantitative Spectroscopy and Radiative Transfer, vol. 200, pp. 1-11, 2017.

[9] C. M. Sorensen, Y. W. Heinson, W. R. Heinson, J. B. Maughan, and A. Chakrabarti, "Q-space analysis of the light scattering phase function of particles with any shape," Atmosphere, vol. 8, no. 4, article 68, 2017.

[10] L. Bi and P. Yang, "Impact of calcification state on the inherent optical properties of Emiliania huxleyi coccoliths and coccolithophores," Journal of Quantitative Spectroscopy and Radiative Transfer, vol. 155, pp. 10-21, 2015.

[11] L. C. Henyey and J. L. Greenstein, "Diffuse radiation in the galaxy," Astro-phys, vol. 93, pp. 70-83, 1941.

[12] J. Farnlund, C. Holman, and P. Kageson, "Emissions of ultrafine particles from different types of light duty vehicles," Tech Rep 10, 2001.

[13] H. R. Pruppacher and J. D. Klett, Microphysics of Clouds and Precipitation, Kluwer Acad., Norwell, Mass, USA, 1978.

[14] Q. Fu, K. N. Liou, M. C. Cribb, T. P. Charlock, and A. Grossman, "Multiple scattering parameterization in thermal infrared radiative transfer," Journal of the Atmospheric Sciences, vol. 54, no. 24, pp. 2799-2812, 1997.

[15] P. Yang and K. N. Liou, "Finite-difference time domain method for light scattering bysmall ice crystals in threedimensional space," J Opt Soc Amer, vol. 13, 1996.

[16] J.-Q. Zhao, G. Shi, H. Che, and G. Cheng, "Approximations of the scattering phase functions of particles," Advances in Atmospheric Sciences, vol. 23, no. 5, pp. 802-808, 2006.

[17] M. I. Mishchenko, L. D. Travis, and D. W. Mackowski, “T-matrix computations of light scattering by nonspherical particles: a review," Journal of Quantitative Spectroscopy and Radiative Transfer, vol. 55, no. 5, pp. 535-575, 1996.

[18] P. Yang and K. N. Liou, "Geometric-optics-integral-equation method for light scattering by nonspherical ice crystals," Applied Optics, vol. 35, no. 33, pp. 6568-6584, 1996.

[19] M. I. Mishchenko, L. D. Travis, R. A. Kahn, and R. A. West, "Modeling phase functions for dustlike tropospheric aerosols using a shape mixture of randomly oriented polydisperse spheroids," Journal of Geophysical Research Atmospheres, vol. 102, no. 14, pp. 16831-16847, 1997.

[20] O. Dubovik, A. Sinyuk, T. Lapyonok et al., "Application of spheroid models to account for aerosol particle nonsphericity in remote sensing of desert dust," Journal of Geophysical Research D: Atmospheres, vol. 111, no. 11, article D11208, 2006.

[21] T. Nakajima, M. Tanaka, M. Yamano, M. Shiobara, K. Arao, and Y. Nakanishi, "Aerosol Optical Characteristics in the Yellow Sand Events Observed in May, 1982 at Nagasaki-Part II Models," Journal of the Meteorological Society of Japan. Ser. II, vol. 67, no. 2, pp. 279-291, 1989.

[22] Q. Fu, T. J. Thorsen, J. Su, J. M. Ge, and J. P. Huang, “Test of Mie-based single-scattering properties of non-spherical dust aerosols in radiative flux calculations," Journal of Quantitative Spectroscopy and Radiative Transfer, vol. 110, no. 14-16, pp. 1640$1653,2009$. 
[23] Z. Meng, P. Yang, G. W. Kattawar, L. Bi, K. N. Liou, and I. Laszlo, "Single-scattering properties of tri-axial ellipsoidal mineral dust aerosols: A database for application to radiative transfer calculations," Journal of Aerosol Science, vol. 41, no. 5, pp. 501-512, 2010.

[24] M. I. Mishchenko and L. D. Travis, "T-matrix computations of light scattering by large spheroidal particles," Optics Communications, vol. 109, no. 1-2, pp. 16-21, 1994.

[25] P. Yang, Q. Feng, G. Hong et al., "Modeling of the scattering and radiative properties of nonspherical dust-like aerosols," Journal of Aerosol Science, vol. 38, no. 10, pp. 995-1014, 2007.

[26] K. N. Liou, Y. Gu, Q. Yue, and G. McFarguhar, "On the correlation between ice water content and ice crystal size and its application to radiative transfer and general circulation models," Geophysical Research Letters, vol. 35, no. 13, article L13805, 2008.

[27] K. N. Liou, Y. Takano, Q. Yue, and P. Yang, "On the radiative forcing of contrail cirrus contaminated by black carbon," Geophysical Research Letters, vol. 40, no. 4, pp. 778-784, 2013.

[28] P. Yang, L. Bi, B. A. Baum et al., "Spectrally consistent scattering, absorption, and polarization properties of atmospheric ice crystals at wavelengths from 0.2 to $100 \mu \mathrm{m}$," Journal of the Atmospheric Sciences, vol. 70, no. 1, pp. 330-347, 2013. 

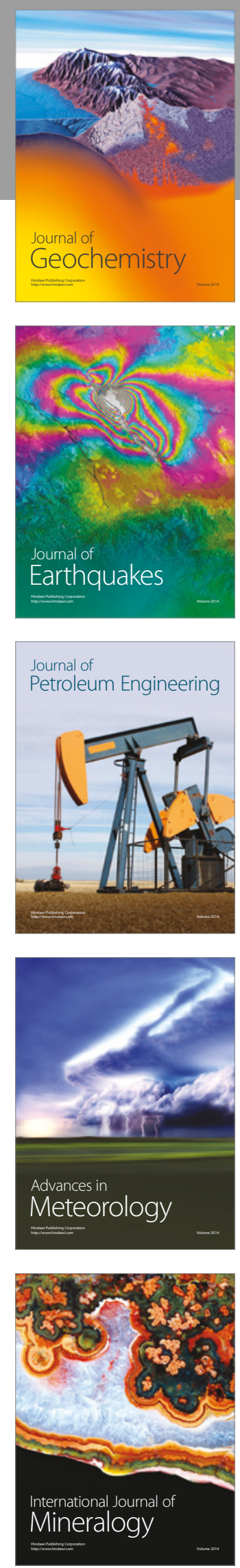
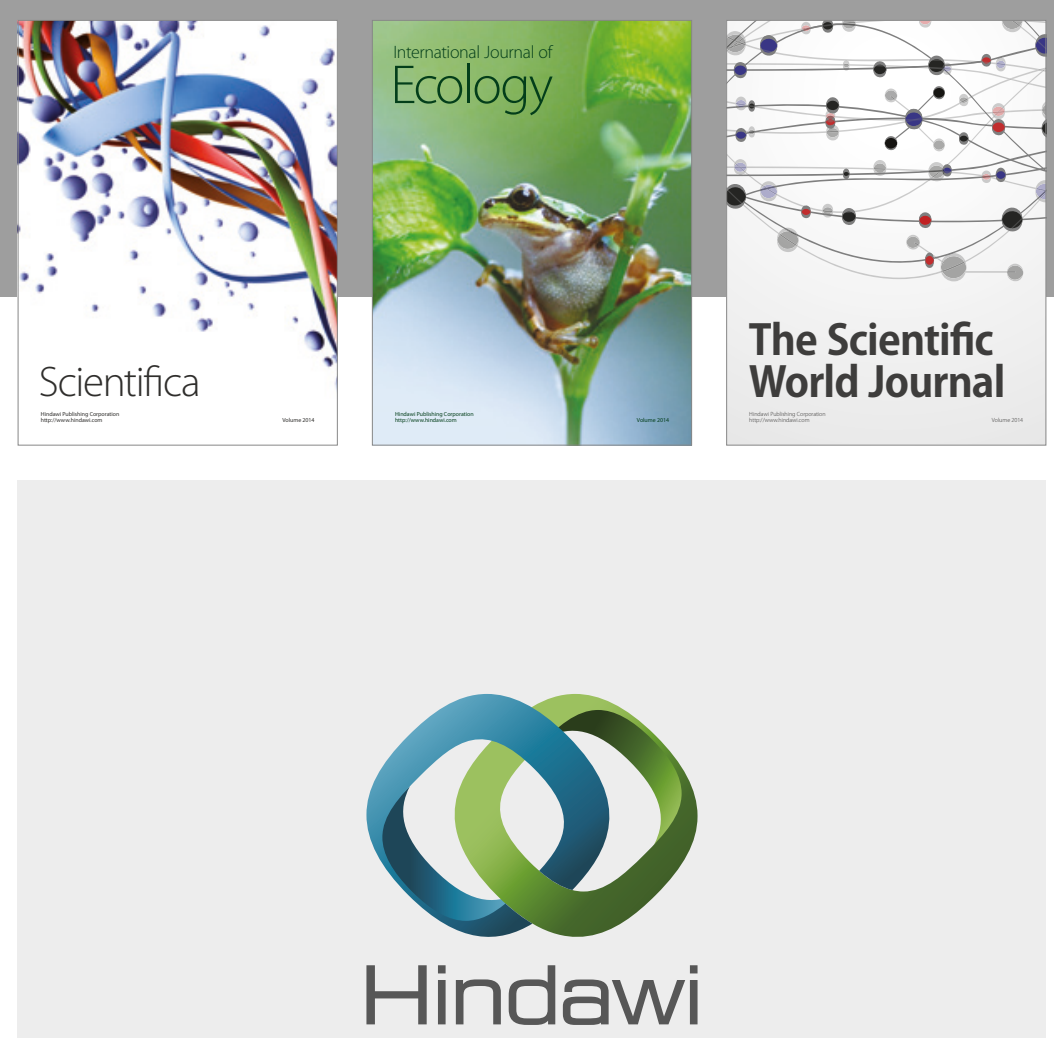

Submit your manuscripts at

https://www.hindawi.com
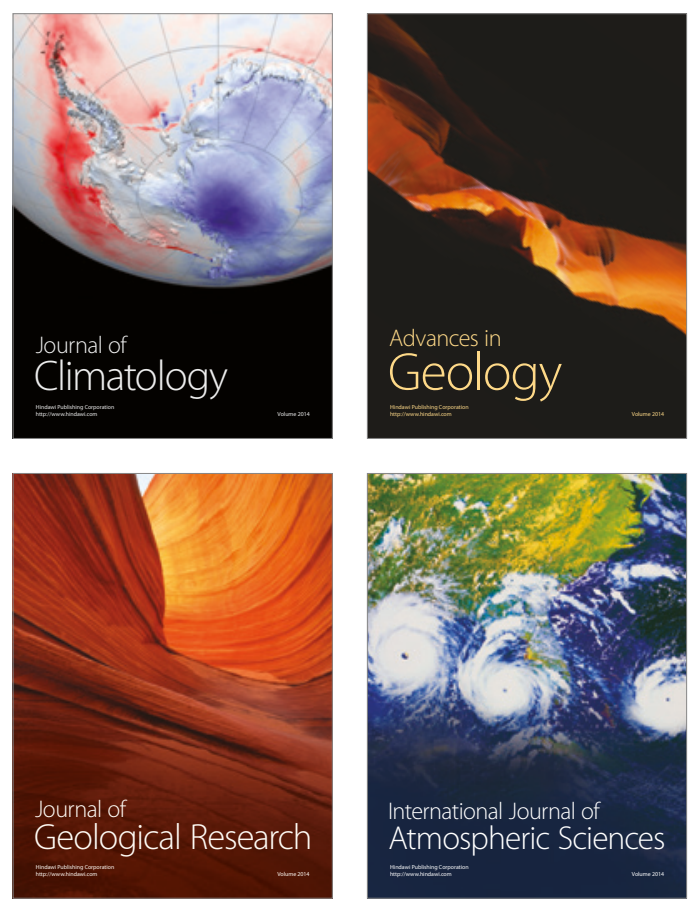

The Scientific

World Journal
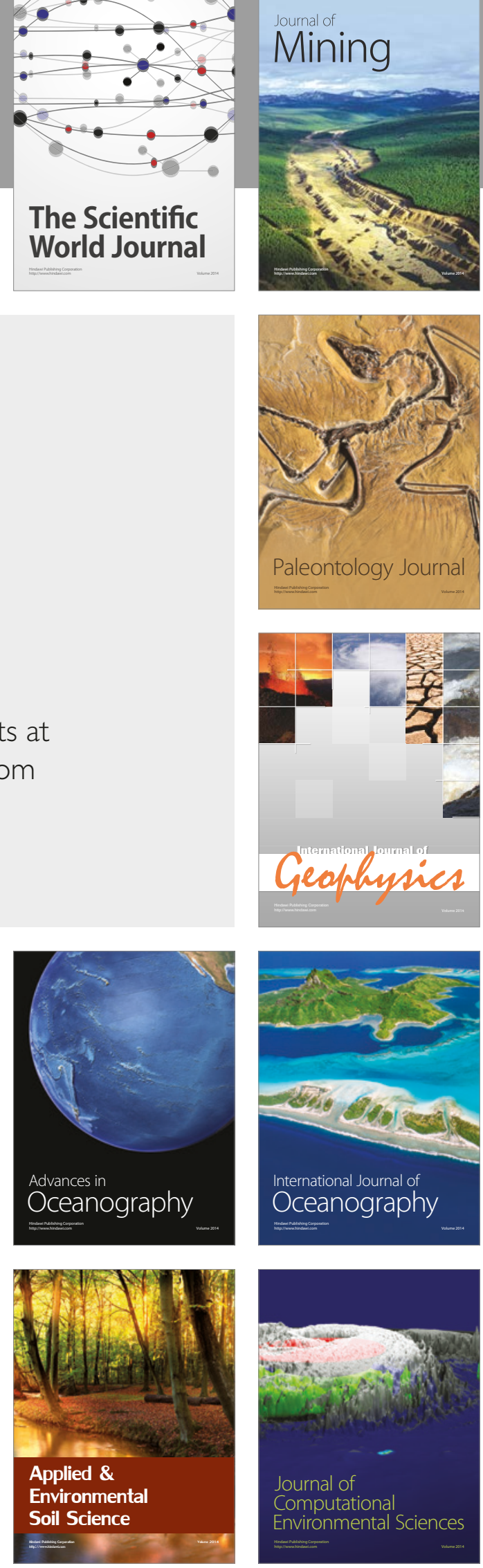